\title{
Flutamida: Revisão de suas propriedades farmacológicas e físico- químicas. Métodos de análise em formulações farmacêuticas
}

Flutamide: a review of its pharmacological and physico-chemical properties. Methods for analysis of pharmaceutical formulas

Hérida Regina Nunes Marona*, Madge Patrícia Betto Storti** e Vicente Alexandre De Lucca Neto***

\begin{abstract}
Resumo
A flutamida, 2-metil-N -[4-nitro-3-(trifluorometil)fenil]propanamida, $\alpha, \alpha, \alpha$-trifluoro-2-metil-4'-nitro-mpropionotoluidida ou 4'-nitro-3'-trifluorometilisobutiranilida, é um fármaco antiandrógeno não-esteróide, destituída de atividade hormonal, utilizada no tratamento do câncer avançado de próstata. A tua inibindo a ligação da testosterona ou da diidrotestosterona aos receptores nucleares e citoplasmáticos nas células-alvo. A flutamida é completamente absorvida pelo trato gastrintestinal, e rapidamente convertida em vários metabólitos. A ligação às proteínas plasmáticas é de $94 \%$ a $96 \%$. 0 fármaco e seus metabólitos são excretados principalmente na urina. As principais reações adversas relacionadas ao uso da flutamida são a ginecomastia e a diarréia leve. Sob a forma de matéria-prima ou em formulações farmacêuticas, a flutamida pode ser determinada por métodos como polarografia, espectrofotometria, cromatografia gasosa e cromatografia líquida de alta eficiência. 0 presente trabalho discorre sobre as propriedades farmacológicas e físico-químicas da flutamida, bem como os métodos de análise aplicados à determinação do fármaco.
\end{abstract}

Palavras-chave: neoplasias prostáticas; flutamida; antiandrógenos; farmacocinética; terapia; efeitos adversos.

\section{Abstract}

Flutamide, 2-metil-N -[4-nitro-3-(trifluorometyl)phenyl]propanamide, $\alpha, \alpha, \alpha$-trifluoro-2-methil-4'-nitro-mpropionotoluidide or 4'-nitro-3'-trifluoromethylisobutyranilide, is a nonsteroidal antiandrogenic drug devoid of hormonal agonist activity, used in treatment of advanced prostate cancer. It inihibits the binding of testosterone or the dihydrotestosterone to nuclear and cytoplasmic receptors in target cells. Flutamide is completely absorbed from the gastrointestinal tract and rapidly converted to a variety of metabolites. Is $94 \%$ to $96 \%$ bound to plasma proteins. The parent drug and metabolites are primarily eliminated by the urine. The principal side effects are gynecomastia and diarrhea. In bulk or pharmaceutical dosage forms, flutamide can be estimated by polarography, spectrophotometry, gas chromatography and high performance liquid chromatography. The present communication reports pharmacological and physico-chemical properties of flutamide and analytical methods for its estimation.

Key words: prostatic neoplasms; flutamide; antiandrogen; pharmacokinetics; therapy; adverse effects.

* D epartamento de Fármacos e M edicamentos - Faculdade de Ciências Farmacêuticas -U N ESP - 14801-902 - Araraquara - SP - Brasil

** Fundação Dr. Amaral Carvalho - 17210080 - Jaú - SP - Brasil

*** Centro de Pesquisa e Pós-graduação - USC - 17011-160 - Bauru - SP - Brasil 


\section{INTRODUÇÃO}

No mundo inteiro, o câncer de próstata ocupa o quinto lugar entre as neoplasias de maior mortalidade ${ }^{1,2}$. $\mathrm{N}$ o Brasil, estima-se que entre os tumores malignos que ocorreram no sexo masculino em 2002, o câncer de próstata teve a segunda taxa mais elevada de mortalidade e a segunda mais elevada de incidência, superado apenas pelo câncer de pele não melanoma ${ }^{3}$.

0 planejamento terapêutico em pacientes com câncer de próstata é feito em função do estágio da doença e do grau de diferenciação histopatológica da neoplasia. N os tumores localizados, a cura pode ser obtida através de cirurgia radical, denominada prostatectomia, ou de radioterapia, e nos tumores disseminados, a paliação e o controle parcial da doença são conseguidos com 0 uso da orquiectomia ou hormonioterapia4.

Em 1941, H uggins e $\mathrm{H}$ odges demonstraram a dependência androgênica dos tumores de próstata, 0 que, posteriormente, determinou como estratégia terapêutica a supressão hormonal através de orquiectomia ou de castração medicinal ${ }^{5}$. 0 bloqueio da testosterona promove a involução substancial do tumor, com significativa melhora dos sintomas clínicos.

As opções de tratamento atual para o carcinoma de próstata em estágio inicial incluem a prostatectomia, a radioterapia e a hormonioterapia ${ }^{6}$.

Atualmente, os tratamentos de câncer de próstata em estágio avançado podem ser realizados pelo decréscimo da produção de androgênio, através da administração de um estrógeno (estrogenoterapia) ou de um análogo do hormônio liberador do hormônio luteinizante (LHRH), como, por exemplo, a leuprolida ou a goserelina, que inibem a atividade testicular por via hipofisária. Emprega-se, também, a administração de um antiandrógeno, como a flutamida, que inibe a penetração ou a ação intracelular de testosterona nas células prostáticas, causando o bloqueio do crescimento do tumor ${ }^{7}$. A associação de flutamida e leuprolida apresentou-se benéfica em pacientes que apresentavam o câncer de próstata8.

Os antiandrógenos podem ser divididos em dois grupos, esteroidais e não-esteroidais, e são compostos utilizados com a finalidade de bloquear a ação dos esteróides androgênios testiculares e adrenais nas células prostáticas, como forma alternativa de supressão endócrina. O s antiandrógenos esteroidais, como 0 acetato de ciproterona, são fármacos que não apenas competem com a testosterona e a diidrotestosterona pela ligação aos receptores andrógenos, mas também exercem ação progestática, inibindo parcialmente a liberação hipofisária de hormônio luteinizante e a produção de testosterona, potencializando o seu efeito antitumoral ${ }^{9,10}$.

A flutamida é um fármaco antiandrógeno nãoesteróide, destituída de atividade hormonal, utilizada no tratamento do câncer avançado de próstata, como monoterapia ou em combinação com outros agentes. Foi o primeiro antiandrogênio não-esteróide, indicado para o tratamento do câncer avançado de próstata a ser comercializado no Brasil ${ }^{10}$.

Sob a forma de matéria-prima ou em formulações farmacêuticas, a flutamida pode ser determinada por métodos como polarografia, espectrofotometria, cromatografia gasosa e cromatografia líquida de alta eficiência.

\section{PROPRIEDADES FARMACOLÓGICAS}

\section{FarmacodinÂMICA}

O s antiandrogênios são compostos desenvolvidos com a finalidade de bloquear a ação de androgênios de origem adrenal e testicular nos tecidos-alvo, sem reduzir os níveis de testosterona. Embora a orquiectomia, os estrógenos ou os agonistas LH RH promovam a redução de $90 \%$ a $95 \%$ na concentração de testosterona no soro, a concentração intraprostática da diidrotestosterona decresce apenas $50 \%$ a $60 \%$, indicando que as adrenais são responsáveis por $40 \%$ a $50 \%$ da diidrotestosterona total na próstata do homem ${ }^{11}$.

A flutamida e seus metabólitos, principalmente a 2-hidróxi-flutamida, atuam inibindo a ligação da testosterona ou da diidrotestosterona aos receptores nucleares e citoplasmáticos nas células-alvo; entretanto, estudos demonstram pequena correlação entre a afinidade relativa de ligação do fármaco aos receptores androgênicos e proliferação, o que sugere que seu efeito benéfico não se deve à interação direta com as células tumorais ${ }^{12}$.

A afinidade da 2-hidróxi-flutamida ao receptor androgênico parece ser 20 vezes maior que a da flutamida?.

\section{Farmacocinética}

A flutamida é completamente absorvida pelo trato gastrintestinal, e rapidamente convertida em vários metabólitos, sendo que o principal é a 2-hidróxiflutamida.

A concentração plasmática máxima da flutamida, 
bem como valores correspondentes da 2-hidróxiflutamida, é atingida em 0,5 a 1,5 h após a administração de dose oral única de $250 \mathrm{mg}$. 0 steady-state é atingido 6 dias após o início da administração de $250 \mathrm{mg}$ de flutamida, 3 vezes ao dia. A ligação às proteínas plasmáticas é de $94 \%$ a $96 \%$ para a flutamida, e 92 a 94\% para a 2-hidróxi-flutamida ${ }^{14}$.

A flutamida e seus metabólitos são excretados principalmente na urina, tendo somente $4,2 \%$ da dose excretada nas fezes após 72 h. 0 metabólito encontrado em maior quantidade na urina é o 2-amino-5-nitro-4(trifluormetil)-fenol. A meia-vida de eliminação é de 4 a 6,6 h após dose oral de $250 \mathrm{mg}$ em pacientes com câncer de próstata, e de 8,1 h em pacientes geriátricos ${ }^{13}$.

\section{TOXICOLOGIA}

As principais reações adversas relacionadas ao uso da flutamida são a ginecomastia e a diarréia leve. $\mathrm{N}$ ão há evidência de interferência nos parâmetros hematológicos ou hormonais. Elevações transitórias nos valores das transaminases hepáticas e da bilirrubina podem ocorrer.

A flutamida não apresenta os numerosos efeitos colaterais que limitam o uso dos antiandrogênios esteróides, tais como a diminuição da libido, retenção hídrica, doenças tromboembólicas e problemas cardiovasculares. A libido e potência sexual são conservadas em $80 \%$ dos pacientes ${ }^{14-18}$.

\section{EFICÁCIA TERAPÊUTICA}

Atualmente, os tratamentos de carcinoma avançado de próstata podem ser realizados por quatro alternativas: - orquiectomia, que é a retirada cirúrgica de parte do testículo que produz o hormônio e que reduz $90 \%$ da testosterona plasmática;

- estrogenoterapia, administração de um estrógeno que diminui o emprego de análogos do LHRH, como, por exemplo, a leuprolida ou a goserelina, que inibem a atividade testicular por via hipofisária;

- emprego de antiandrogênicos periféricos, como a flutamida, que inibem o bloqueio do crescimento do tumor, pela penetração ou a ação intracelular de testosterona nas células prostáticas?.

D esde o início da década de 1950, os urologistas têm conhecimento que o carcinoma de próstata responde à manipulação hormonal pela castração cirúrgica ou pelo uso de dietilestilbestrol. A pesar de 50 anos de experiência com a terapia hormonal, ainda existem controvérsias em relação à duração do tratamento, à prática de monoterapia ou à utilização de antiandrógeno combinado ao uso de terapia hormonal intermitente, ou à combinação de terapia hormonal com quimioterapia ${ }^{19}$.

Estudos comparando a monoterapia androgênica com a orquiectomia, dietilestilbestrol e agonistas do hormônio liberador do hormônio luteinizante, demonstram taxas de sobrevida e eficácia similares. A monoterapia com antiandrogênios é usualmente bem tolerada, com exceção da alta incidência de ginecomastia (34 a 100\%), a qual, entretanto, é infreqüente quando a flutamida é usada em combinação com ambos os métodos de castração ${ }^{18}$.

Em estudo multicêntrico realizado por Crawford et al. ${ }^{8}$, foi observado que o bloqueio androgênico completo (agonista LHRH + flutamida) foi mais efetivo do que a monoterapia com um agonista LHRH. N este caso, a terapia combinada aumentou o tempo de sobrevida livre da progressão da doença, assim como a duração média da sobrevida. Estudos recentes revelaram que a taxa de sobrevivência num período de 5 anos pelo uso do bloqueio androgênico máximo seria em torno de 2\% a 3\% superior, se comparada à supressão androgênica isolada ${ }^{20}$.

$\mathrm{H}$ á relatos também do uso da flutamida no tratamento da acne e do hirsutismo em mulheres ${ }^{21-22}$, e no tratamento da hiperplasia prostática benigna ${ }^{23}$.

\section{PROPRIEDADES FÍSICO-QUÍMICAS}

\section{DESCRIÇ̃̃o}

A flutamida, quimicamente denominada 2-metil-N [4-nitro-3-(trifluorometil)fenil]propanamida, 4'-nitro-3'trifluorometilisobutiranilida ou $\alpha, \alpha, \alpha$-trifluoro-2-metil4'-nitro-m-propionotoluidida ${ }^{24}$ (Figura 1), apresenta-se como pó cristalino amarelado, insolúvel em água, mas livremente solúvel em solventes orgânicos polares, como acetona, etanol, dimetilsulfóxido, acetato de etila, metanol e polietilenoglicol 400, e solúvel em clorofórmio, éter e propilenoglicol25-27. Apresenta fórmula molecular $\mathrm{C}_{11} \mathrm{H}_{11} \mathrm{~F}_{3} \mathrm{~N}_{2} \mathrm{O}_{3}(\mathrm{C}=47,83 \% ; \mathrm{H}=$ $4,02 \% ; N=10,14 \% ; F=20,63 \%$ e $\mathrm{O}=17,38 \%$ ) e massa molar igual a $276,22 \mathrm{~g} / \mathrm{mol}$. 
Figura 1 - Estrutura química da flutamida.<smiles>CC(C)C(=O)Nc1ccc([N+](=O)[O-])c(C(F)(F)F)c1</smiles>

SÍNTESE

O s métodos gerais descritos para a síntese da flutamida (Figura 2) são a reação de condensação, de para-nitração e a geração de cloreto ácido in situ ${ }^{28}$.

Figura 2 - Síntese da flutamida por reações de condensação, para-nitração e formação de cloreto ácido em situ.<smiles>CC(=O)Nc1cccc(C(F)(F)F)c1</smiles>

$\mathrm{N}$ a reação de condensação, cloreto de isobutanoíla é adicionado lentamente, sob agitação, a uma solução resfriada de 4-nitro-3-trifluorometilanilina em piridina. A mistura reacional é, então, aquecida em banho de vapor e, posteriormente, resfriada em banho de gelo e filtrada. 0 sólido é lavado com água e recristalizado em benzeno.

Um método alternativo é a reação de para-nitração, a qual consiste na adição lenta de ácido nítrico $90 \%$, sob agitação a $5^{\circ} \mathrm{C}$, a m-trifluorometilisobutilanilida. A mistura é, então, filtrada e o produto formado é seco, após lavagem com água para remover o excesso de ácido.

0 utro processo muito utilizado é a reação de cloreto de tionila em dimetilacetamida (DM A), a $-20^{\circ} \mathrm{C}$, para formar cloreto ácido in situ, o qual reage com 4-nitro3-trifluorometilanilina para produzir a flutamida.

As principais impurezas encontradas na flutamida ${ }^{26}$ (Figura 3) são a 4-nitro-3-(trifluorometil)anilina (a), N -[4-nitro-3-(trifluorometil)fenil]acetamida(b), 3-(trifluorometil)anilina (c), N -[4-nitro-3-(trifluorometil)fenil]propanamida(d), 2-metil$\mathrm{N}$-[4-nitro-3-(trifluorometil)fenil]propanamida(e) eN -[2-nitro5-(trifluorometil)fenil]propanamida (f).

Figura 3 - Principais impurezas encontradas na flutamida.<smiles>Nc1ccc([N+](=O)[O-])c(C(F)(F)F)c1</smiles>

(a)<smiles>Nc1cccc(C(F)(F)F)c1</smiles>

(c)

(e)<smiles>CC(C)C(=O)Nc1ccc([N+](=O)[O-])c(C(F)(F)F)c1</smiles><smiles>CC(=O)Nc1ccc([N+](=O)[O-])c(C(F)(F)F)c1</smiles>

(b)<smiles>CCOC(=O)Nc1ccc([N+](=O)[O-])c(C(F)(F)F)c1</smiles>

(d) (f) 
mineral, com o espectro padrão. Alternativamente, a identificação pode ser realizada através da comparação do tempo de retenção do pico principal do cromatograma em $C L A E$, com o pico principal do padrão de referência USP.

0 espectro da flutamida na região do visível/UV apresenta absorção máxima em $380 \mathrm{~nm}$.

Vários métodos espectrofotométricos foram descritos para a determinação de flutamida em preparações farmacêuticas e na matéria-prima. Um método de determinação por espectrofotometria na região do visível, utilizando a formação de um composto colorido quando a flutamida é dissolvida em $\mathrm{HCl}$, foi proposto por Zarapkar et al. ${ }^{31}$. 0 cromógeno, estável por uma hora, apresenta absortância máxima em 380 nm. A lei de Beer é observada na faixa de concentração entre 2,5 e $15,0 \mu \mathrm{g} / \mathrm{mL}$. 0 método não sofre interferência de excipientes comuns, como amido e talco, e os baixos valores do desvio padrão e coeficiente de variação indicam a sua precisão e reprodutibilidade.

$\mathrm{N}$ agaraja et al. ${ }^{32}$ desenvolveram um método baseado na interação do fármaco, na sua forma reduzida, com 0 reagente dicloridrato de $\mathrm{N}$-naftiletilenodiamina em meio neutro, ou com o resorcinol em meio alcalino. A absortância dos cromóforos, estáveis por sete dias, foi medida a 525 e $480 \mathrm{~nm}$, respectivamente. A lei de Beer é observada na faixa de concentração entre 0,1 e 7,0 $\mu \mathrm{g} / \mathrm{mL}$. 0 método não sofre interferência dos excipientes comumente utilizados nas formulações de flutamida, porém, outras aminas apresentam reação positiva nas condições utilizadas na reação de diazotação. Parâmetros estatísticos e estudos de recuperação foram aplicados para demonstrar a precisão e repetibilidade do método.

M urthy et al. ${ }^{33}$ utilizaram método espectrofotométrico baseado na oxidação do fármaco com excesso conhecido de permanganato de potássio. A quantidade remanescente de permanganato de potássio foi determinada através da sua capacidade de oxidar 0 corante Fast Green FCF, sendo que o decréscimo na intensidade da cor da solução, proporcional à concentração da flutamida, foi medido em $625 \mathrm{~nm}$. A faixa de concentração em que o método obedece a lei de Beer situa-se entre 0,2 e 1,6 $\mu \mathrm{g} / \mathrm{mL}$. 0 s excipientes comumente utilizados em formulações farmacêuticas não interferem no método, o qual foi validado com estudos de recuperação. 0 método aplica-se também à determinação de meloxicam e nimessulida, podendo ser aplicado à matéria-prima ou formulações farmacêuticas.

Reddy et al. ${ }^{34}$ desenvolveram dois métodos para a quantificação da flutamida em formulaçõesfarmacêuticas ou em matéria-prima, baseados na reação do fármaco reduzido com 0 ácido cromotrópico (método $A$ ), com formação de cromógeno medido em $525 \mathrm{~nm}$, e com 0 resorcinol (método $B$ ), cujo cromógeno apresenta absorvância máxima em $485 \mathrm{~nm}$. A lei de Beer é obedecida entre 1,0 e $8,0 \mu \mathrm{g} / \mathrm{mL}$ para o método $A$ e 1,0 e $5,0 \mu \mathrm{g} / \mathrm{mL}$ para o método $\mathrm{B}$. O s resultados obtidos foram comparados com método de referência e estudos de recuperação confirmaram a sensibilidade e repetibilidade dos métodos propostos.

$R$ angappa et al. ${ }^{35}$ empregaram o cloridrato de prometazina na presença de $\mathrm{N}$-bromosuccinamida como reagente para a determinação da flutamida matéria-prima e em preparações farmacêuticas. 0 método exige processo extrativo com clorofórmio e o cromóforo, estável por quatro dias em temperatura de $25 \pm 3^{\circ} \mathrm{C}$, foi medido em $505 \mathrm{~nm}$. 0 método obedece a lei de Beer na faixa de 0,25 a 15,0 $\mu \mathrm{g} / \mathrm{mL}$, não sofre interferência de excipientes e teve a sua precisão avaliada através de estudos de recuperação e análise estatística dos resultados.

0 utros métodos descritos para a determinação da flutamida incluem a espectrofotometria na região do ultravioleta ${ }^{35}$, a polarografia ${ }^{36-38}$, a cromatografia gasosa ${ }^{39}$ e CLAE ${ }^{40}$.

Alguns métodos foram descritos para a análise do fármaco em fluidos corporais e tecidos, dentre os quais podemos citar mid-bore cromatografia ${ }^{41}$ e CLAE ${ }^{42}$.

\section{Agradecimentos: Ao CN Pq.}

\section{REFERÊNCIAS BIBLIOGRÁFICAS}

1. Pisani P, Parkin D M , Bray F, Ferlay J. Estimates of the worldwidemortality from 25 cancers in 1990 [published erratum appearsin Int J C ancer 1999 D ec 10;83(6):8703]. Int J Cancer 1999;83(1):18-29.

2. Ferlay J, Parkin D M , Pisani P. G lobocan 1: cancer incidence and mortality worldwide. Lyon: IARC; 1998.

3. M inistério daSaúde. SecretariadeAssistênciaàSaúde Instituto $\mathrm{N}$ acional de $\mathrm{C}$ âncer (Brasil). Estimativas da incidência e mortalidadepor câncer. Rio dejaneiro: IN CA; 2002.

4. Srougi M, Dzik C. Câncer de próstata. In: Sociedade Brasileira de $U$ rologia. $M$ anual de normatização: câncer urológico. São Paulo: BG Cultural; 2000. p. 33-41.

5. H uggins $C, H$ odges $C V$. Studies on prostatic cancer I: the effect of castration, of estrogen and of androgen injection on serum phosphatasesin metastatic carcinoma of theprostate. Cancer Res 1941;1:293-7.

6. M oul JW, Anderson J, Penson D F, K lotzLH, Soloway M S, Schulman CC. Early prostatecancer: prevention, treatment modalities, and quality of life issues. Eur U rol 2003;44(3):283-93.

7. Pratt $W B$, Ruddon, RW, Ensminger WD, M aybaum J. Anticancer D rugs. 2nd ed. N ew York: $O$ xford University; 1994. 
8. C rawford ED, Eisenberger M A, M CL eod D G, Spaulding JT, Benson R, D orr FA, et al. A controlled trial of leuprolide with and without flutamide in prostatic carcinoma [published erratum appears in N Engl J M ed $1989 \mathrm{~N}$ ov 16;321(20):1420]. N Engl J M ed 1989;321(7):419-24.

9. SociedadeBrasileira deU rologia. Tumores prostáticos. In: Concenso Brasileiro deC âncer dePróstata, 1. 1998.

10. Srougi M . Câncer de próstata. In: Srougi M , Simon SD . Câncer urológico. São Paulo: Platina; 1990. p. 281-347.

11. LabrieF, D upontA, Belanger A. Completeandrogen blockade for the treatment of prostate cancer. In: D eVita VT, $\mathrm{H}$ ellman S, Rosenberg AS. Important advances in oncology. Philadelphia: JB Lippincott; 1985. p. 193-217.

12. Brodgen RN, Chrisp P. Flutamide: a review of its pharmacodinamic and pharmacokinetic properties, and therapeutic usein advanced prostatic cancer. D rugs Aging 1991;1(2):104-15.

13. Radwanski E, Perentesis G, Symchowicz S, Zampaglione $\mathrm{N}$. Singleand multipledosepharmacokinetic evaluation of flutamidein normal geriatric volunteers. J C lin Pharmacol 1989;29(6):554-8.

14. Balducci L, Parker $M$, H escock $H$, Tantranond $P$, Sexton W. Systemic management of prostatecancer. Am J M ed Sci 1990;299(3):185-92.

15. Brogden RN , Clissold SP. Flutamide: a preliminary review of its pharmacodynamic and pharmacokinetic properties, and therapeutic efficacy in advanced prostatic cancer. $D$ rugs 1989;38(2):185-203.

16. D elaere KP, Van Thillo EL. Flutamide monotherapy as primary treatment in advanced prostatic carcinoma. Semin Oncology 1991;18(6):13-8.

17. Boccon-Gibod L. Arenon-steroidal anti-androgen appropriate as monotherapy in advanced prostate cancer? Eur U rology 1998;33(2):159-64.

18. Schroder FH . Antiandrogensas monotherapy for prostate cancer. Eur Urology 1998;34(3):12-7.

19. RichieJP. Anti-androgens and other hormonal therapies for prostate cancer. U rology 1999;54:15-8.

20. Dalesio 0 , van Tinteren $H$, Clarke M, Peto R, Schroder $\mathrm{FH}, \mathrm{D}$ echering I, et al. $\mathrm{M}$ aximum androgen blockade in advanced prostatecancer: an overview of the randomised trials. Lancet 2000;355(9214):1491-8.

21. Shaw JC. Antiandrogen and hormonal treatment of acne. Dermatol Clin 1996;14(4)803-11.

22. Young R, Sinclair R. H irsutes II: treatment. Austr J D ermatol 1998;39(3):151-7.

23. Jonler M , Riehmann M , Bruskewitz RC. Benign prostatic hyperplasia. D rugs 1994;47(1):66-81.

24. TheM erck Index. 13th ed. WhitehouseStation:TheIndex M erck; 2001.

25. The U nited States Pharmacopeia. 25th ed. Rockville: U nited States Pharmacopeial Convention; 2002.
26. British Pharmacopoeia. London:T heStationery O ffice; 2001.

27. European Pharmacopoeia. 3rd ed. Strasbourg: C ouncil of Europe; 2001.

28. Sternal R, N ugara N . Flutamide. Anal ProfilesD rug Subst Exc 2001;27:115-57.

29. Cense JM, Agafonov V, Ceolin R, Ladure P, Rodier N . Crystal and molecular structure analysis of flutamide. Bifurcated helicoidal $\mathrm{C}-\mathrm{H}$...O hydrogen bonds. Struct Chem 1994;5(2):79-84.

30. C eolin R, AgafonovV, G onthierVassal A, Szwarc H, Cense JM , LadureP. Solid-statestudies on crystallineand glassy flutamidethermodynamic evidence for dimorphism. J Thermal Anal 1995;45(6):1277-84.

31. Zarapkar SS, D amle CD , H alkar U P. Spectrophotometric determination of flutamideand its pharmaceutical formuIation. Indian D rugs 1996;33(5):193-4.

32. N agaraja P, SunithaKR, Silwadi M F. N ew spectrophotometric method for the determination of flutamidein pharmaceutical preparations. J Pharm Biomed Anal 2000;23:617-22.

33. M urthyTK, Reddy M N , Reddy M D , Sankar D G. Spectrophotometric determination of flutamida, nimesulideand meloxicam. Asian J Chem 2001;13(3):915-8.

34. Reddy M N , M urthyTK, Reddy M D , Sankar D G. Spectrophotometric estimation of flutamide in pharmaceutical dosageforms. Asian J Chem 2001;13(3):1261-2.

35. RangappaKS, N agarajaP, M urthy KCS. N ew extractivespectrophotometric determination of flutamidein pureand pharmaceutical formulations. Analytical Sci 2000;16:637-9.

36. Alvarez-LuejeA, Pena C, N unez-Vergara LJ, SquellajÁ. Electrochemical study of flutamide, an anticancer drug, and its polarographic, UV spectrophotometric and H PLC determination in tablets. Electroanalysis 1998;10(15):1043-51.

37. Snycerski A. Polarographic determination of flutamide. J Pharm Biomed Anal 1989;7(12):1513-8.

38. Snycerski A, Kalinowski M K. Eletrochemical properties of flutamide, a new anticancer drug. Polish J Chem 1992;66(1):49-52.

39. Sane RT, G angrade M G, Bapat VV, Sure SR. G as chromatographic determination of flutamide, nimodipineand ticlopidinehydrochloridefrom their pharmaceutical preparations. Indian D rugs 1993;30:147-50.

40. H endershot S, Koharski D . Appropiatecolumn configurationsfor therapid analysisand semipreparativepurification of theradiolabeled drug flutamideby high-performanceliquid chromatography. J C hrom A 2001;914:23-7.

41. Farthing D , SicaD , Fakhry I, Walters D L, C efali EA, Allan $G$. D etermination of flutamide and hydroxyflutamidein dog plasma by a sensitivehigh performanceliquid chromatography method utilizing mid-bore chromatography. Biomed Chromatogr 1994;8(5):251-4. 
42. Leibinger M K. N ew and validated high-performance liquid chromatographic method for determination of hydroxyflutamidein human plasma.J Pharm Biomed Anal 1996;14:1377-81. 